Annick Fontbonne 1 Eduardo Freese-de-Carvalho 1,2 Moab Duarte Acioli 3 Geisa Amorim de Sá 4 Eduarda Angela Pessoa Cesse 1

\section{Fatores de risco para poliparasitismo intestinal em uma comunidade indígena de Pernambuco, Brasil}

\author{
Risk factors for multiple intestinal parasites \\ in an indigenous community of the State \\ of Pernambuco, Brazil
}

1 Departamento de Saúde Coletiva, Núcleo de Estudos em Saúde Coletiva, Centro de Pesquisas Aggeu Magalhães, Fundação Oswaldo Cruz.

Rua dos Coelhos 450, Recife, PE 50070-550, Brasil.

2 Departamento de Medicina Social, Universidade Federal de Pernambuco. Av. Morais Rêgo s/no, Cidade Universitária, Recife, PE 50670-901, Brasil.

3 Faculdade de Filosofia do Recife. Av. Conde da Boa Vista, 921 Recife, PE 50060-002, Brasil. 4 Fundação Nacional de Saúde. Av. Conselheiro Rosa e Silva 1489, Recife, $P E$ 52050-020, Brasil. nesc@cpqam.fiocruz.br

\begin{abstract}
An investigation into the ethno-epidemiological profile of the Pankararu indigenous group in the State of Pernambuco, Brazil, identified multiple intestinal parasites in nearly all members of the community. To detect possible environmental risk factors, we used the data base from a previous survey to test relations between daily living conditions (housing, sanitation, water supply and treatment, and garbage disposal) and the number of different parasite species found in the same household. The sample consisted of 84 families from the original sample of 112. Selection was based on the number of stool tests performed in the family. The mean number of parasite species was 5.0 per family, for a mean family size of 6.1 members. This number was greater for wattle-and-daub houses (mean 6.0 parasite species $v$ s. 4.9 for brick houses; $p<0.03$ ) and when water used in the household was not treated (mean 5.1 parasite species, vs. 4.5 for treated water; $p<0.05)$. Other household characteristics and hygienic habits did not significantly influence this number. We concluded that multiple intestinal parasitism in the Pernambuco Pankararu community is frequent, to the point of being the rule, and that it relates essentially to water source and treatment.
\end{abstract}

Key words Epidemiology; Intestinal Parasitism; Parasites; Pankararu; South American Indians

Resumo No perfil etnoepidemiológico da comunidade dos Índios Pankararus - interior do Estado de Pernambuco -, as parasitoses intestinais representam importante problema de saúde pública, por atingir a quase totalidade da população. A fim de conhecer possíveis fatores de risco ambientais deste quadro, utilizou-se parte da base de dados do inquérito original para relacionar as condições de moradia ao número de parasitas diferentes verificado entre seus moradores. Com base na seleção da quantidade de exames coprológicos efetuados entre as pessoas da família, a amostra para análise contou 84 famílias dentre as 112 da amostra aleatória original. Para o número médio de 6,1 pessoas por família, constatou-se que o de parasitas diferentes presentes no lar era 5,0, número crescente quando a casa era de taipa (6,0 contra 4,9 para as de alvenaria; $p<0,03)$, ou a água usada na moradia não era tratada (5,1 contra 4,5 para água tratada; $p<0,05)$. Outros fatores que caracterizam a moradia e sua higiene não parecem influenciar o número médio de parasitas na casa. Conclui-se que o poliparasitismo nos Índios Pankararus de Pernambuco chega a representar a regra e está referido sobretudo às fontes de água de beber e ao seu tratamento. Palavras-chave Epidemiologia; Parasitoses Intestinais; Parasitos; Pankararu; Índios Sul-Americanos 


\section{Introdução}

Apesar de poucos países coletarem e analisarem rotineiramente estatísticas vitais ou estatísticas dos serviços de saúde por grupo étnico, é sabido que há sérias desigualdades no que se refere à saúde e à assistência de saúde das populações indígenas das Américas (PAHO, 1997). A fim de combater eficazmente e prevenir os agravos à saúde que acometem estas populações, faz-se necessário conhecer melhor tanto as características do perfil epidemiológico como as particularidades do modo de vida, seja do ponto de vista das condições materiais de vida, seja do conjunto das práticas histórico-culturais.

No Brasil, a estruturação e o aperfeiçoamento do Programa de Saúde do Índio - do Ministério da Saúde (MS) / Fundação Nacional de Saúde (FNS), sob a coordenação da COSAI (Coordenação de Saúde do Índio) - vêm ampliando esse trabalho em parceria intersetorial e interinstitucional no âmbito federal e estadual. Este Programa subsidiou, em fins de 1996, um grande inquérito para a avaliação do perfil etnoepidemiológico da comunidade dos Índios Pankararu, no Sertão de Pernambuco. O componente epidemiológico incluía um exame coprológico, cujos resultados demostraram altíssima prevalência de parasitoses intestinais (Freese-de-Carvalho \& Acioli, 1997), fato relativamente incomum entre as populações rurais do interior de Pernambuco - em particular, do Agreste e Sertão (Perez et al., 1999). Segundo estes autores verificaram, em duas vilas não indígenas do Sertão existia ausência de parasitismo: em $64 \%$ da amostra em Cajueiro e de $48 \%$ em Caatingueira; por sua vez, no Agreste do Estado, em Inajeira, a ausência de parasitismo foi de $60 \%$ da amostra, não havendo praticamente poliparasitismo. Nesse contexto, em razão do achado surpreendente na tribo Pankararu, o presente artigo define, como objetivo, a identificação de alguns dos possíveis fatores de risco ambientais associados a esse problema.

\section{Métodos}

O inquérito supracitado foi conduzido na área indígena pertencente à tribo dos Pankararu, situada no Sertão do São Francisco, Pernambuco, a 412 quilômetros da capital do Estado (Figura 1). Foram escolhidas cinco aldeias - dentre as 12 existentes na área indígena -, por terem maior número de habitantes, correspondendo a cerca de $70 \%$ da população total da tribo. As aldeias escolhidas são: Brejo dos Padres, Saco dos Barros, Espinheiro, Tapera e Serrinha.
O componente epidemiológico do inquérito foi conduzido em amostra aleatória de 112 domicílios ("famílias"), representando $20 \%$ das 550 famílias residentes nas cinco aldeias escolhidas. O sorteio das residências foi baseado em listagem do Inquérito Sanitário realizado em maio de 1996 pela FNS. Preferencialmente, o chefe de cada família foi entrevistado para fornecer informações acerca das características da moradia (construção, saneamento, destino do lixo e número de pessoas que compartilham o domicílio), da morbidade referida dos membros da família, da utilização dos serviços de saúde - incluindo a utilização de práticas tradicionais de cura -, além de informações de cunho sócio-econômico. A entrevista era complementada pela realização de parasitológico das fezes, pelo método de sedimentação, para todos os membros da família, por meio de técnica especializada do laboratório da FNS de Pernambuco, deslocada para o campo.

Dois arquivos informatizados foram constituídos para armazenar os dados do inquérito: o primeiro para os dados da família - ou seja, obtidos através da entrevista de um de seus membros - e o segundo, para os resultados individuais dos exames complementares. A presente análise - tendo como objetivo a identificação de fatores de risco ambientais para a alta prevalência de parasitoses intestinais descoberta quando do inquérito-fonte - foi feita mediante abordagem ecológica, tendo a família como unidade de estudo. Em conseqüência, os dados dos exames coprológicos, obtidos ao nível individual, foram aglomerados por família, de modo a representar mais uma de suas características, que poderíamos chamar de "estado parasitológico da família”. As variáveis criadas para quantificar tal estado são as seguintes: o número de espécies diferentes de parasitas encontradas entre todos os membros da família que tiveram exame coprológico ("número de parasitas"); a razão deste número pelo número de membros da família que efetuaram exame coprológico ("número de parasitas por pessoa"); e a proporção de exames coprológicos positivos para uma dada espécie de parasita, entre todos os exames coprológicos realizados na família ("proporção de pessoas infestadas na família”).

Nem todas as famílias do inquérito-fonte foram estudadas na presente análise, em virtude da impossibilidade de construir variáveis ecológicas confiáveis em famílias de tamanho reduzido e/ou em que poucos membros tiveram exame coprológico. Os seguintes critérios foram aplicados para excluir famílias da amostra finalmente analisada: quando nenhum membro tinha exame coprológico (18 famílias); quan- 

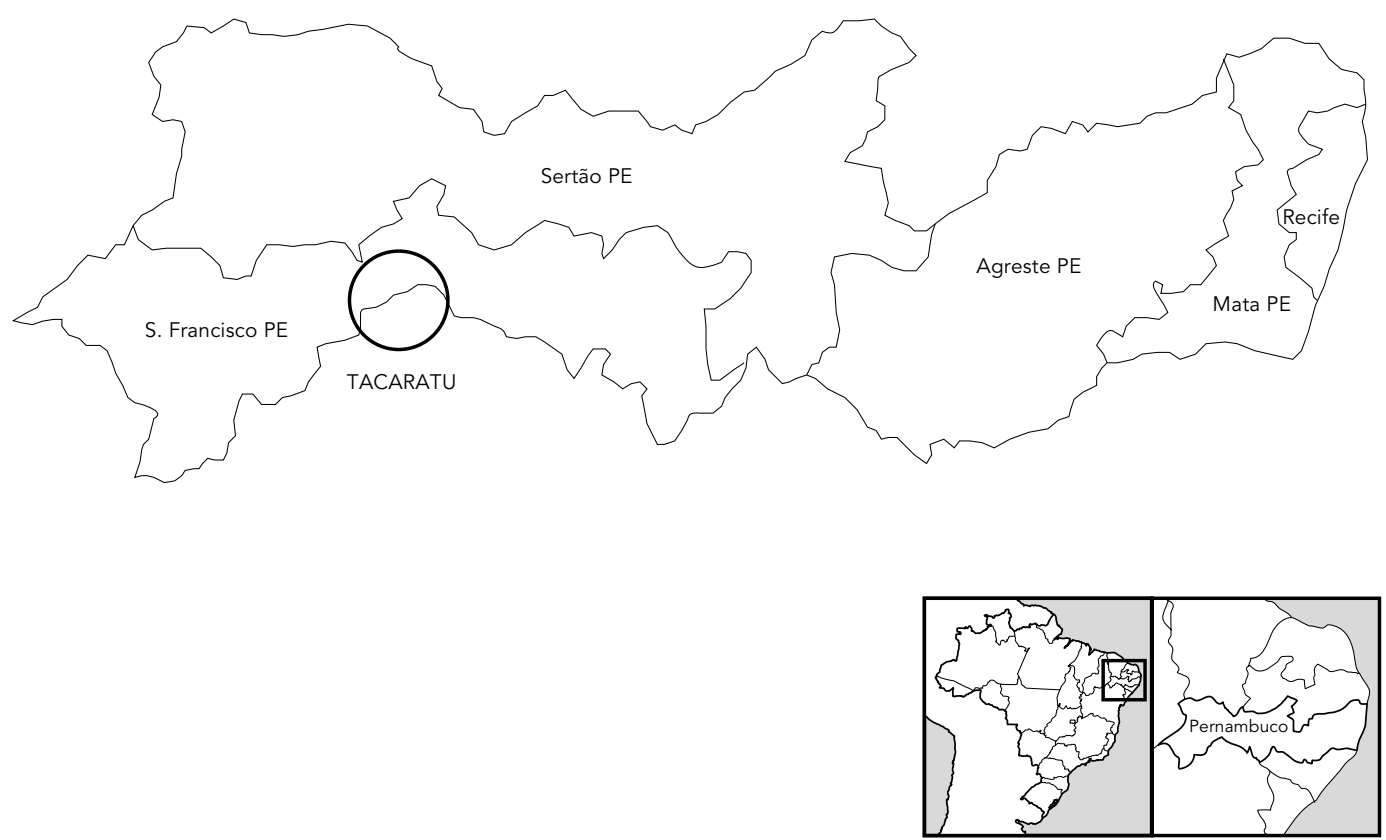

do somente um membro tinha exame coprológico (oito famílias); quando menos da metade dos membros da família tinha exame coprológico (duas famílias). Resultou que 84 famílias foram incluídas na presente análise.

As variáveis independentes - ou seja, testadas enquanto possíveis fatores de risco do "estado parasitológico da família" - foram extraídas diretamente do arquivo que continha os dados da família. A análise buscou relações entre estas variáveis e as variáveis dependentes, quantificando o "estado parasitológico da família", descritas antes, utilizando os testes paramétricos de comparação de médias (t de Student e análise de variância) do pacote estatístico SPSS (SPSS Incorporation, 1995). Diferenças de médias que geraram valor de $\mathrm{p}$ menor que 0,05 foram consideradas estatisticamente significativas. O termo "desvio-padrão" foi abreviado como DP.

\section{Resultados}

As 84 famílias selecionadas totalizavam 510 pessoas, ou seja, tamanho médio de 6,1 pessoas por família. A maioria das famílias (84,5\%)
Tabela 1

Composição da amostra, por idade e sexo.*

\begin{tabular}{lrrr} 
& Sexo masculino & Sexo feminino & Total (\%) \\
\hline 0-4 anos & 32 & 24 & $56(12,1 \%)$ \\
$5-9$ anos & 37 & 35 & $72(15,6 \%)$ \\
$10-14$ anos & 38 & 25 & $63(13,6 \%)$ \\
$\geq 15$ anos & 129 & 142 & $271(58,7 \%)$ \\
Total $(\%)$ & $236(51,1 \%)$ & $226(48,9 \%)$ & 462 \\
\hline
\end{tabular}

* Não informados: 48 pessoas.

era de tipo nuclear, apesar de contar, com freqüência, mais de duas gerações juntas. A composição por idade e sexo encontra-se detalhada na Tabela 1.

Exames coprológicos foram realizados em 417 pessoas. Ficou evidente o fato de o poliparasitismo ser regra na comunidade dos Pankararus. Em todas as famílias havia, pelo menos, um membro parasitado. Ademais, três foi o número mínimo de espécies de parasitas diferentes presentes na mesma casa, encontrando-se o máximo de nove e a média de cinco (Figura 
2). A proporção de pessoas parasitadas dentro do mesmo domicílio variava de acordo com a espécie de parasitas. Verificamos (Tabela 2), como espécie mais freqüente, a Entamoeba histolytica, que infestava em média $82,4 \%$ dos habitantes da mesma casa. Esta parasitose atingia quase a totalidade dos adultos $(95,3 \%)$ e $40,7 \%$ das crianças de 0 a 4 anos de idade. O segundo parasita mais freqüente era o Ascaris lumbricoides, que infestava, em média, 51,2\% dos moradores - em especial, as crianças abaixo de 15 anos. Nesta faixa etária, foi grande, também, a proporção de infestados por Giardia lamblia $(62,0 \%)$.

O número de espécies diferentes presentes na mesma família elevava-se, logicamente, com o tamanho dessa família (Tabela 3). Da mesma maneira, esse número crescia se a família habitava casa de taipa e não de alvenaria; se a fonte da água não destinada para beber era do reservatório e não de outras fontes (cacimba ou barreiro); e se a água usada na casa não era tratada, ou seja, nem fervida nem filtrada. As outras características da casa (piso, existência de banheiro dentro ou fora de casa, conexão à rede elétrica e destino do lixo) não mostraram relação significativa com o número de espécies diferentes de parasitas presentes nas famílias (Tabela 4).

Pelo fato de existir relação entre o número de espécies diferentes de parasitas e o tamanho da família, testamos a possibilidade de

Figura 2

Distribuição de freqüência do número de espécies diferentes de parasitas presentes na mesma família.

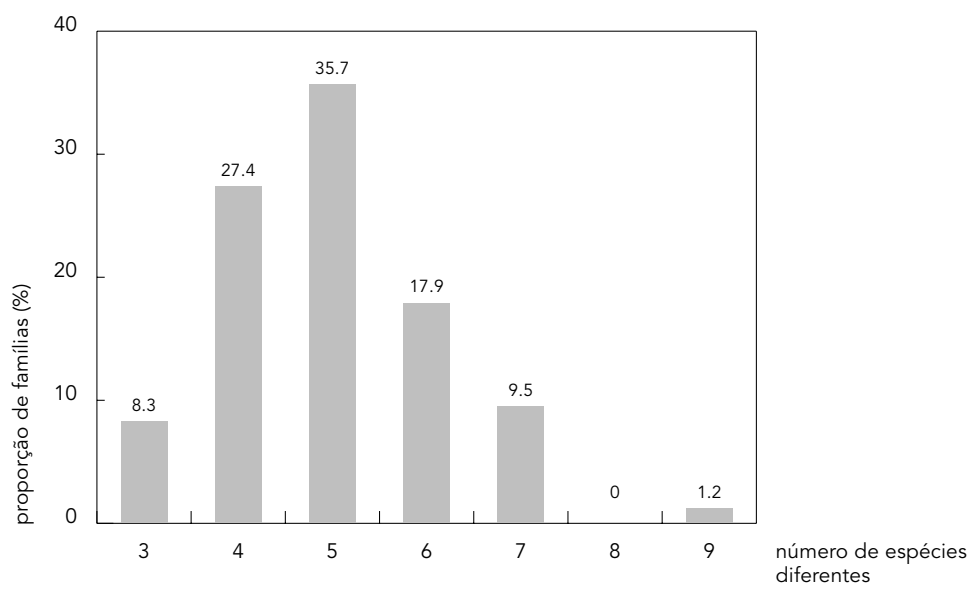

este último introduzir um fator de confusão. Comprovamos a ausência de relação significativa entre o tamanho da família e as características da casa - listadas na Tabela 4 -, exceto nos casos de "fonte de água para beber" e "fonte de água para outros usos", em que observamos que as famílias usuárias do reservatório tinham significativamente mais membros que as outras: em média, 6,5 membros contra 4,6 (água de beber) ou 4,7 (água para outros usos) nas famílias que não usavam o reservatório (diferença significativa, $\mathrm{p}<0,01)$. Diante desta diferença, decidimos ajustar a comparação do número de espécies de parasitas segundo o uso ou não do reservatório, criando a variável "número de (espécies de) parasitas por pessoa”. Com esta variável, a dessemelhança passou a ser significativa na outra direção, quando se comparava com o teste não ajustado - ou seja, nas famílias que usavam água do reservatório havia menos espécies diferentes de parasitas por pessoa. Em média, encontrava-se 0,86 parasita por pessoa (DP: 0,26) nas famílias que usavam o reservatório como fonte de água de beber contra 1,02 (DP: 0,36) naquelas que não o usavam ( $\mathrm{p}<0,03)$; e 0,84 parasita por pessoa (DP: $0,24)$ nas famílias que recorriam ao reservatório como fonte de água para outros usos contra 1,08 (DP: 0,38) nas que não o usavam ( $\mathrm{p}<0,001$ ).

\section{Discussão}

Nossa análise tem limitações; contudo, a mais importante diz respeito ao caráter ecológico, que não permitiu determinar os fatores de risco para poliparasitismo em cada indivíduo. Por construção, a variável dependente principal (número de espécies diferentes presentes em cada casa) pode resumir situações bem diferentes, que não pudemos especificar e que podem apresentar fatores de risco diversos. Por exemplo, um número de três espécies dentro da mesma família pode significar que três membros são atingidos com uma espécie diferente cada um, ou que somente um membro está infestado por três parasitas. No entanto, este tipo de análise é apropriado para evidenciar os fatores de risco que procurávamos - ou seja, fatores de risco do ambiente familiar relacionados com a moradia e com os principais hábitos de higiene praticados nela. Todavia, vale ressaltar que, devido ao forte caráter de coletivização observado dentro das aldeias, a probabilidade de infestar-se fora do ambiente doméstico é grande, o que pode ter diminuído a potência para descobrir outros fatores de risco próprios da moradia. 
Proporção média de pessoas infestadas, por idade, segundo a espécie de parasita.

\begin{tabular}{lccccc}
\hline Espécie & $\mathbf{0 - 4}$ anos (\%) & $\mathbf{5 - 9}$ anos (\%) & $\mathbf{1 0 - 1 4}$ anos (\%) & $\mathbf{1 5}$ anos (\%) & Total (\%) \\
\hline Entamoeba histolytica & 40,7 & 62,9 & 90,0 & 95,3 & 82,4 \\
Ascaris lumbricoides & 79,6 & 84,3 & 73,3 & 26,2 & 51,2 \\
Escherichia coli & 18,5 & 31,4 & 48,3 & 63,9 & 50,3 \\
Giardia lamblia & 83,3 & 71,4 & 31,7 & 3,4 & 29,2 \\
Ancilostomídeos & 9,3 & 22,9 & 36,7 & 6,9 & 23,6 \\
lodamoeba butschilli & 1,9 & 1,4 & 6,7 & 6,0 & 5,9 \\
Trichuris trichiura & 3,7 & 0 & 6,7 & 1,7 & 5,1 \\
Strongyloides stercoralis & 0 & 2,9 & 6,7 & 0,9 & 2,5 \\
Taenia saginata & 1,9 & 5,7 & & 2,1 \\
\hline
\end{tabular}

Com esta análise, conseguimos mostrar a prevalência altíssima de infestação por parasitas e a freqüência do poliparasitismo na comunidade dos Pankararu. Nenhuma família está poupada: há, no mínimo, três parasitas diferentes dentro de cada lar, conformando a média de cinco, para um tamanho médio de seis membros por família. Isto sugere, em combinação com a análise da proporção de moradores infestados (Tabela 2), que a grande maioria das pessoas está infestada por uma espécie de parasita ao menos. Estes números assustadores estão entre os mais altos descritos em comunidades indígenas, confrontando-se somente com relatórios mais antigos (Coimbra Jr. \& Mello, 1981; Lawrence et al., 1983). Os inquéritos mais recentes têm encontrado, em geral, baixas prevalências e raridade do poliparasitismo (Ferrari et al., 1992; Santos et al., 1995), apesar de um artigo (Miranda et al., 1998) apontar para números muito semelhantes aos nossos também em termos de tipo de parasitas mais freqüentes - em uma tribo do Estado do Pará.

Outro elemento importante é que as espécies mais comuns que infestam os Pankararu são de importância epidemiológica e clínica. Vale destacar que a amebíase por Entamoeba histolytica - doença com sérias conseqüências potenciais para a saúde - é a mais freqüente das parasitoses intestinais nessa população. Tal resultado só foi encontrado na tribo amazonense, conforme o artigo acima mencionado (Miranda et al., 1998). O Ascaris lumbricoides, parasita comum - em especial, nas crianças só chega a ocupar o segundo lugar. Isso é possível que tenha ocorrido porque a população da tribo foi medicalizada com mebendazol no último ano antes do inquérito. Outras parasitoses graves - como: giardíase e ancilostomíase -
Tabela 3

Relação entre o tamanho da família e o número de espécies diferentes de parasitas encontradas nela.

\begin{tabular}{lc}
\hline $\mathbf{n}$ o de espécies diferentes & no médio ( \\
\hline 3 & $4,0 \pm 0,8$ \\
4 & $5,3 \pm 1,7$ \\
5 & $6,1 \pm 2,2$ \\
6 & $7,1 \pm 1,8$ \\
7 ou mais & $7,8 \pm 2,5$ \\
Total $(5,0 \pm 1,2)$ & $6,1 \pm 2,2$ \\
\hline
\end{tabular}

* $p<0,01$ para diferença das médias (análise de variância).

afetam em torno de um quarto dos indivíduos. Fora das complicações específicas a cada espécie, um parasitismo desta importância traz conseqüências gerais para o estado de saúde, como a má nutrição e a anemia (Ferreira et al., 1998; Tshikuka et al., 1997). O problema de saúde pública, assim delineado, fica evidente.

Nossa análise procurou focalizar os fatores de risco do aumento do poliparasitismo familiar. Fora das comunidades indígenas, a infestação por enteroparasitas é claramente relacionada com a pobreza e a precariedade das condições de higiene e saneamento (Coimbra Jr. \& Santos, 1991; Machado et al., 1996; TavaresDias \& Grandini, 1999). Acontece que a pobreza, bem como precárias condições de higiene e saneamento, caracteriza o conjunto da comunidade Pankararu do sertão de Pernambuco. No entanto, são esses mesmos fatores que aparecem como relevantes, dentro da própria comunidade, para separar menor ou maior risco de poliparasitismo na família. Por exemplo, o 
Relação entre o número de espécies diferentes de parasitas por família e as variáveis que caracterizam a casa.

\begin{tabular}{|c|c|c|c|}
\hline \multirow{2}{*}{$\begin{array}{l}\text { Variável } \\
\text { Paredes }\end{array}$} & \multicolumn{2}{|c|}{ no médio ( \pm DP) de espécies diferentes de parasita } & \multirow{2}{*}{$\begin{array}{c}\text { Valor de } p \\
\quad<0,03\end{array}$} \\
\hline & $\begin{array}{c}\text { Alvenaria }(n=78) \\
4,9 \pm 1,1\end{array}$ & $\begin{array}{c}\text { Taipa }(n=6) \\
6,0 \pm 1,1\end{array}$ & \\
\hline Piso & $\begin{array}{c}\text { Cimento }(n=68) \\
4,9 \pm 1,1\end{array}$ & $\begin{array}{l}\text { Terra batida }(n=16) \\
5,3 \pm 1,3\end{array}$ & NS* \\
\hline Banheiro & $\begin{array}{c}\operatorname{Sim}(n=15) \\
5,1 \pm 1,2\end{array}$ & $\begin{array}{l}\text { Não }(n=69) \\
5,0 \pm 1,2\end{array}$ & NS \\
\hline Rede elétrica & $\begin{array}{c}\text { Conectado }(n=71) \\
5,0 \pm 1,2\end{array}$ & $\begin{array}{c}\text { Não conectado }(n=13) \\
4,8 \pm 1,2\end{array}$ & NS \\
\hline Fonte de água de beber & $\begin{array}{c}\text { Reservatório }(n=65) \\
5,1 \pm 1,2\end{array}$ & $\begin{array}{l}\text { Outra }(n=19) \\
4,6 \pm 1,1\end{array}$ & NS \\
\hline Fonte de água para outros usos & $\begin{array}{c}\text { Reservatório }(n=65) \\
5,1 \pm 1,2\end{array}$ & $\begin{array}{l}\text { Outra }(n=19) \\
\quad 4,5 \pm 0,9\end{array}$ & $<0,04$ \\
\hline Tratamento da água & $\begin{array}{c}\operatorname{Sim}(n=19) \\
4,5 \pm 1,0\end{array}$ & $\begin{array}{c}\text { Não }(n=64) \\
5,1 \pm 1,3\end{array}$ & $<0,05$ \\
\hline Destino do lixo & $\begin{array}{l}\text { Queimado ou enterrado }(n=36) \\
\qquad 4,8 \pm 1,2\end{array}$ & $\begin{array}{c}\text { Céu aberto }(n=48) \\
5,1 \pm 1,1\end{array}$ & NS \\
\hline
\end{tabular}

* NS = não significativo.

papel da pobreza é verificado pelo fato de o tipo de casa (taipa ou alvenaria) influenciar o número de espécies diferentes que acometem os moradores, ressaltando-se que a casa de taipa indica grau de pobreza ainda maior nessa comunidade, a qual é globalmente muito desfavorecida. Da mesma forma, variáveis relacionadas com a água e o seu tratamento destacaram-se como fatores de risco para poliparasitismo ainda mais acentuado. De maneira geral, as fontes de água mais usadas na comunidade não apresentam condições de segurança sanitária. Por exemplo, os reservatórios são precários e sem tampa de proteção. Mesmo assim, as poucas famílias $(n=19)$ que recorrem a tratamento - ainda que muitas vezes imperfeito mediante fervura ou filtragem da água que consomem, exibem menores taxas de poliparasitismo em comparação com as famílias que ora não tratam a água por nenhum método, ora recorrem ao método tradicional de "coar" a água em pano limpo, prática que obedece a crenças locais de "purificação", mas que não tem demostrado eficácia na luta contra contaminações. Antigas tradições, como a de coar a água, podem ter tido alguma relevância no passado. Hoje em dia, certo grau de sedentarismo e a alta prevalência de parasitas que circulam nas aldeias obrigam a contestar o método como forma de prevenir as enteroparasitoses, exemplificando a necessária complementaridade das visões epidemiológica e antropológica para elaborar modelos de intervenção que melhorem a saúde das populações indígenas de nosso país.

Este último achado indica as particularidades que devem guiar a organização e a forma do atendimento às populações indígenas do Brasil (Confalonieri, 1989).

Os resultados deste trabalho apontam para precárias condições de saneamento nas comunidades Pankararu investigadas, que resultam não somente em elevados níveis de parasitismo intestinais, como também na freqüente ocorrência de poliparasistismo. Tais achados corroboram que os povos indígenas constituem um segmento socioeconomicamente bastante desfavorecido no Brasil, o qual, em larga medida, está excluído das principais medidas públicas que podem melhorar as condições de vida e de saúde, tais como: saneamento ou abastecimento de água apropriada para consumo humano. 


\section{Agradecimentos}

O presente trabalho baseia-se na análise de parte dos dados coletados quando do inquérito "Avaliação do Perfil Etnoepidemiológico de uma Comunidade Indígena do Estado de Pernambuco", patrocinado pela Fundação Nacional de Saúde/CODEPRO (Coordenação de Projetos)/Centro Nacional de Epidemiologia/ COSAI (Coordenação de Saúde do Índio) e FUNAI (Fundação Nacional de assistência ao Índio), com recursos do Banco Mundial.

\section{Referências}

COIMBRA Jr., C. E. A. \& MELLO, D. A., 1981. Enteroparasitas e Capillaria sp. entre o grupo Suruí, Parque Indígena Aripuanã, Rondônia. Memórias do Instituto Oswaldo Cruz, 76:299-302.

COIMBRA Jr., C. E. A. \& SANTOS, R. V., 1991. Avaliação do estado nutricional num contexto de mudança sócio-econômica: O grupo indígena Suruí do Estado de Rondônia, Brasil. Cadernos de Saúde Pública, 7:538-562.

CONFALONIERI, U. E. C., 1989. O Sistema Único de Saúde e as populações indígenas: Por uma integração diferenciada. Cadernos de Saúde Pública, 5:424-440.

FERRARI, J. O.; FERREIRA, M. U.; CAMARGO, L. M. \& FERREIRA, C. S., 1992. Intestinal parasites among Karitiana Indians from Rondônia State, Brazil. Revista do Instituto de Medicina Tropical de São Paulo, 34:223-225.

FERREIRA, M. R.; SOUZA, W.; PEREZ, E. P.; LAPA, T.; CARVALHO, A. B.; FURTADO, A. F.; COUTINHO, H. B. \& WAKELIN, D., 1998. Intestinal helminthiasis and anaemia in youngsters from Matriz da Luz, district of São Lourenço da Mata, State of Pernambuco, Brazil. Memórias do Instituto Oswaldo Cruz, 93:289-293.

FREESE-DE-CARVALHO, E. \& ACIOLI, M. D., 1997. Avaliação do Perfil Etnoepidemiológico de uma Comunidade Indígena do Estado de Pernambuco. Relatório Final de Pesquisa, Recife: Departamento de Saúde Coletiva, Núcleo de Estudos em Saúde Coletiva, Instituto Aggeu Magalhães, Fundação Oswaldo Cruz.

LAWRENCE, D. N.; NEEL, J. V.; ABADIE, S. H.; MOORE, L. L.; ADAMS, L. J.; HEALY, G. R. \& KAGAN, I. G., 1983. Estudos epidemiológicos entre populações ameríndias da Amazônia. Parasitoses intestinais em povoações recentemente contactadas e em aculturação. Acta Amazonica, 13:393-407.

MACHADO, M. T.; MACHADO, T. M.; YOSHIKAE, R. M.; SCHMIDT, A. L. A.; FARIA, R. C. A.; PASCHOALOTTI, M. A.; BARATA, R. C. B. \& CHIEFFI, P. P., 1996. Ascariasis in the subdistrict of Cavacos, municipality of Alterosa (MG), Brazil: Effect of mass treatment with albendazole on the intensity of infection. Revista do Instituto de Medicina Tropical de São Paulo, 38:265-271.
MIRANDA, R. A.; XAVIER, F. B. \& MENEZES, R. C., 1998. Parasitismo intestinal em uma aldeia indígena Parakanã, Sudeste do Estado do Pará, Brasil. Cadernos de Saúde Pública, 14:507-511.

PAHO (Pan American Health Organization), 1997. Health of indigenous people. Revista Panamericana de Salud Pública, 2:357-362.

PEREZ, E.; GAZIN, P.; FURTADO, A.; MIRANDA, P.; MARQUES, N. M.; SILVA, M. R. \& VARELA, R., 2000. Parasitoses intestinales et schistosomose em milieu urbain, en région littorale et en région semi-aride du Nordeste du Brésil. Cahiers de Santé, 10:127-129.

SANTOS, R. V.; COIMBRA Jr., C. E. A.; FLOWERS, N. M. \& SILVA, J. P., 1995. Intestinal parasitism in the Xavante Indians, Central Brazil. Revista do Instituto de Medicina Tropical de São Paulo, 37:145148.

SPSS INCORPORATION, 1995. SPSS for Windows. Statistical Package for the Social Sciences. Release 7.0. Chicago: SPSS Inc.

TAVARES-DIAS, M. \& GRANDINI, A. A., 1999. Prevalência e aspectos epidemiológicos de enteroparasitoses na população de São José da Bela Vista, São Paulo. Revista da Sociedade Brasileira de Medicina Tropical, 32:63-65.

TSHIKUKA, J. G.; GRAY-DONALD, K.; SCOTT, M. \& OLEKA, K. N., 1997. Relationship of childhood protein-energy malnutrition and parasite infections in an urban African setting. Tropical Medicine and International Health, 2:374-382. 